\title{
Exact Traveling Wave Solutions of a KPI Equation
}

\author{
Yuzhong Zhang \\ School of Information Technology and Engineering, Yuxi Normal \\ University, Yuxi, Yunnan, 653100, China
}

Copyright (C) 2014 Yuzhong Zhang. This is an open access article distributed under the Creative Commons Attribution License, which permits unrestricted use, distribution, and reproduction in any medium, provided the original work is properly cited.

\begin{abstract}
In this paper, the traveling waves of the a KPI equation be investigated. These traveling waves contain periodic blow-up waves, periodic waves, blow-up wave and solitary wave. Under fixed parameter conditions, their exact expressions are given.
\end{abstract}

Keywords: Kadomtsev-Petviashvili equation,periodic blow-up waves,periodic waves, blow-up wave,solitary wave

\section{Introduction and main results}

The well known Kadomtsev-Petviashvili (KP) equation [1]

$\left(u_{t}+6 u u_{x}+u_{x x x}\right)_{x}+3 \kappa u_{y y}-3 u_{z z}=0$.

with $\kappa= \pm 1$, is a universal model for the propagation of weakly nonlinear dispersive long waves which are essentially one directional, with weak transverse effects. When $\kappa=-1$, Eq. (1.1) is usually called KPI, while for $\kappa=1$, it is usually called KPII. The KP equation also arises naturally in many other applications, particularly in plasma physics, gas dynamics, and elsewhere.

Khalfallah [2] has applied the homogeneous balance method to look for exact solutions of the KPI. Sinelshchikov [3] demonstrate that four solutions from 13 of the KPI obtained by Khalfallah [2] are wrong and do not satisfy the equation. In this paper, we employ the method of dynamical systems [4-7] to investigate the traveling waves of the KPI.

In order to state our main results conveniently, for given constant $c$, let 
$f_{0}(\varphi)=\varphi^{3}-\frac{1}{2}(6+c) \varphi^{2}-g \varphi$,

$f(\varphi)=-f_{0}(\varphi)+h$,

$g_{0}=-\frac{(6+c)^{2}}{12}$,

and

$\varphi_{ \pm}=\frac{6+c \pm \sqrt{(6+c)^{2}+12 g}}{6}$.

The $\varphi_{1}$ is a simple real root, $\varphi_{2}$ and $\overline{\varphi_{2}}$ are pair of conjugate complex roots of $f(\varphi)=0$, where $g \leq g_{0}$, or $g>g_{0}$ and $h>f_{0}\left(\varphi_{-}\right)$, or $g>g_{0}$ and $h<f_{0}\left(\varphi_{+}\right)$. the $\varphi_{1}, \varphi_{2}$ and $\varphi_{3}$ are three simple real roots of $f(\varphi)=0$, where $g>g_{0}$ and $f_{0}\left(\varphi_{+}\right)<h<f_{0}\left(\varphi_{-}\right)$.

Using the notations above, our main results are stated in the following Proposition.

Proposition. (1) If $g \leq g_{0}$, or $g>g_{0}$ and $h>f_{0}\left(\varphi_{-}\right)$, or $g>g_{0}$ and $h<f_{0}\left(\varphi_{+}\right)$, then KPI has a periodic blow-up wave solution $u_{1}(x, y, z, t)=\varphi(\xi)$, the $\varphi(\xi)$ has expression as follows

$\varphi=\varphi_{1}+A-\frac{2 A}{1-\operatorname{cn}(\sqrt{2 A} \xi, k)}$,

where $A=\sqrt{\left(\frac{\varphi_{2}+\overline{\varphi_{2}}}{2}-\varphi_{1}\right)^{2}-\frac{\left(\varphi_{2}-\overline{\varphi_{2}}\right)^{2}}{4}}, k=\sqrt{\frac{2\left(A+\varphi_{1}\right)-\left(\varphi_{2}+\overline{\varphi_{2}}\right)}{4 A}}$ is the modulus of Jacobian elliptic function.

(2) If $g>g_{0}$ and $h=f_{0}\left(\varphi_{-}\right)$, then KPI has a blow up wave solution $u_{2}(x, y, z, t)=\varphi(\xi)$ and a solitary wave solution $u_{3}(x, y, z, t)=\varphi(\xi)$, the $\varphi(\xi)$ has expression as follows respectively

$\varphi=\varphi_{-}-\left(\varphi_{1}-\varphi_{-}\right) \sinh ^{-2}\left(\frac{\sqrt{2\left(\varphi_{1}-\varphi_{-}\right)}}{2} \xi\right)$,

and

$\varphi=\varphi_{-}+\left(\varphi_{1}-\varphi_{-}\right) \cosh ^{-2}\left(\frac{\sqrt{2\left(\varphi_{1}-\varphi_{-}\right)}}{2} \xi\right)$.

(3) If $g>g_{0}$ and $f_{0}\left(\varphi_{+}\right)<h<f_{0}\left(\varphi_{-}\right)$, then KPI has a periodic blow up wave solution $u_{4}(x, y, z, t)=\varphi(\xi)$ and a periodic wave solution $u_{5}(x, y, z, t)=\varphi(\xi)$, the $\varphi(\xi)$ has expression as follows respectively

$\varphi=\varphi_{3}-\left(\varphi_{3}-\varphi_{1}\right) \operatorname{sn}^{-2}\left(\frac{\sqrt{2\left(\varphi_{3}-\varphi_{1}\right)}}{2} \xi, k\right)$,

and

$\varphi=\varphi_{3}-\left(\varphi_{3}-\varphi_{2}\right) \operatorname{sn}^{2}\left(\frac{\sqrt{2\left(\varphi_{3}-\varphi_{1}\right)}}{2} \xi, k\right)$,

where $k=\sqrt{\frac{\varphi_{3}-\varphi_{2}}{\varphi_{3}-\varphi_{1}}}$ is the modulus of Jacobian elliptic function.

(4) If $g>g_{0}$ and $h=f_{0}\left(\varphi_{+}\right)$, then KPI has a periodic blow-up wave solution $u_{6}(x, y, z, t)=\varphi(\xi)$, the $\varphi(\xi)$ has expression as follows $\varphi=\varphi_{+}-\left(\varphi_{+}-\varphi_{1}\right) \csc ^{2}\left(\frac{\sqrt{2\left(\varphi_{+}-\varphi_{1}\right)}}{2} \xi\right)$.

Example. Letting $c=1$, then $g_{0} \approx-4.08333$. Letting $g=-3$, then 
$\varphi_{-} \approx 0.565741$ and $\varphi_{+} \approx 1.76759$. Thus $f_{0}\left(\varphi_{-}\right) \approx 0.758076$ and $f_{0}\left(\varphi_{+}\right) \approx-0.109927$.

Taking $h=-1$, we have $\varphi_{1} \approx-0.253156, \varphi_{2} \approx 1.87658-0.654667 i$ and $\overline{\varphi_{2}} \approx 1.87658+0.654667 i$. Thus $A \approx 2.22808$ and $k \approx 0.148561$. Substituting these data into (1.6), on $\xi-u$ plane we draw a periodic blow up wave graph as Fig.1 (a)

Taking $h=f_{0}\left(\varphi_{+}\right) \approx-0.109927$, we have $\varphi_{1} \approx-0.0351838$ and $\varphi_{+} \approx 1.76759$. Substituting these data into (1.11), on $\xi-u$ plane we draw a periodic blow up wave graph as Fig.1 (b).

Taking $h=0.5$, we have $\varphi_{1} \approx 0.219224, \varphi_{2}=1$ and $\varphi_{3} \approx 2.28078$. Thus $k \approx 0.788205$. Substituting these data into (1.9) and(1.10), on $\xi-u$ plane we draw a periodic blow up wave graph as Fig.1 (c) and a periodic wave graph as Fig.1 (d).

Taking $h=f_{0}\left(\varphi_{-}\right) \approx 0.758076$, we have $\varphi_{-} \approx 0.565741$ and $\varphi_{1} \approx 2.36852$. Substituting these data into (1.7) and (1.8), on $\xi-u$ plane we draw a blow up wave graph as Fig.1 (e) and a solitary wave graph as Fig.1 (f).

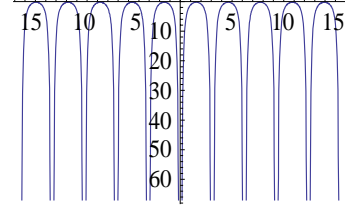

(a)

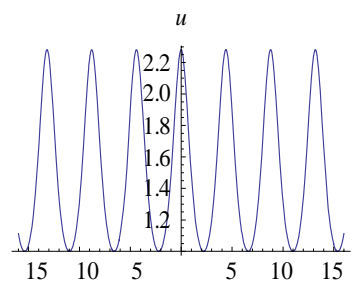

(d)

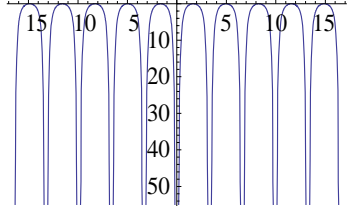

(b)

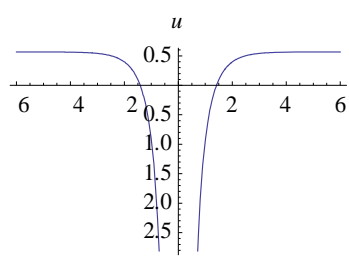

(e)

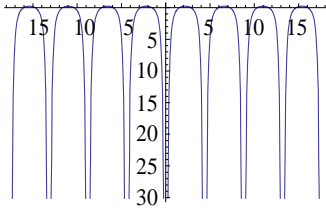

(c)

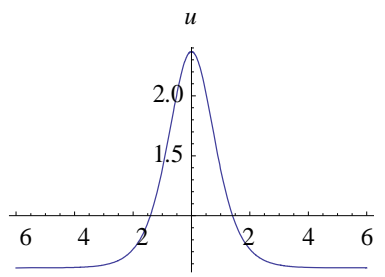

(f)

Fig.1. The periodic blow-up wave, periodic wave, blow up wave and solitary wave of Eq. (1.1) with $c=1$ and $g=-3$. (a). $h=-1$, (b). $h=f_{0}\left(\varphi_{+}\right)$, (c) $h=0.5$, (d). $h=0.5$, (e). $h=f_{0}\left(\varphi_{-}\right)$, (f) $h=f_{0}\left(\varphi_{-}\right)$.

\section{Preliminary}

For given constant $c$, substituting $\xi=x+y+z-c t$ and $u(x, y, z, t)=\varphi(\xi)$ in KPI, it follows that

$\left(-c \varphi^{\prime}+6 \varphi \varphi^{\prime}+\varphi^{\prime \prime \prime}\right)^{\prime}-6 \varphi^{\prime \prime}=0$. 
Integrating (2.1) twice with respect to $\xi$, we have the following traveling wave equation

$\varphi^{\prime \prime}+3 \varphi^{2}-(6+c) \varphi=g$,

where $g$ is integral constant.

In (2.2) multiply that by $\varphi^{\prime}$ and integrating it, we have

$\frac{1}{2}\left(\varphi^{\prime}\right)^{2}=-\varphi^{3}+\frac{1}{2}(6+c) \varphi^{2}+g \varphi+h$,

that is

$\frac{1}{2}\left(\varphi^{\prime}\right)^{2}=f(\varphi)$,

where $h$ is integration constant.

From (1.2), we have

$f_{0}^{\prime}(\varphi)=3 \varphi^{2}-(6+c) \varphi-g$.

Clearly, $f_{0}(\varphi)$ has two extreme points $\varphi_{ \pm}$when $g>g_{0}$, and $f_{0}\left(\varphi_{-}\right)$is maximum, $f_{0}\left(\varphi_{+}\right)$is minimum, has not extreme point when $g \leq g_{0}(c)$. Thus, we have the following results:

(1) $f(\varphi)=-\left(\varphi-\varphi_{1}\right)\left(\varphi-\varphi_{2}\right)\left(\varphi-\overline{\varphi_{2}}\right)$, when $g \leq g_{0}$, or $g>g_{0}$ and $h>f_{0}\left(\varphi_{-}\right)$, or $g>g_{0}$ and $h<f_{0}\left(\varphi_{+}\right)$, where $\varphi_{2}$ and $\overline{\varphi_{2}}$ are pair of conjugate complex.

(2) $f(\varphi)=-\left(\varphi-\varphi_{-}\right)^{2}\left(\varphi-\varphi_{1}\right)$, when $h=f_{0}\left(\varphi_{-}\right)$, where $\varphi_{-}<\varphi_{1}$.

(3) $f(\varphi)=-\left(\varphi-\varphi_{1}\right)\left(\varphi-\varphi_{2}\right)\left(\varphi-\varphi_{3}\right)$, when $f_{0}\left(\varphi_{+}\right)<h<f_{0}\left(\varphi_{-}\right)$, where $\varphi_{1}<\varphi_{2}<\varphi_{3}$.

(4) $f(\varphi)=-\left(\varphi-\varphi_{1}\right)\left(\varphi-\varphi_{+}\right)^{2}$, when $h=f_{0}\left(\varphi_{+}\right)$, where $\varphi_{1}<\varphi_{+}$.

\section{The proof of main results}

(1). When $f(\varphi)=-\left(\varphi-\varphi_{1}\right)\left(\varphi-\varphi_{2}\right)\left(\varphi-\overline{\varphi_{2}}\right)$ and $\varphi \leq \varphi_{1}$, then $f(\varphi) \geq 0$.

Substituting $f(\varphi)$ into (2.4) and integrating it, we have

$\int_{-\infty}^{\varphi} \frac{1}{\sqrt{\left(\varphi_{1}-t\right)\left(t-\varphi_{2}\right)\left(t-\overline{\varphi_{2}}\right)}} d t=\int_{0}^{\xi} \sqrt{2} d t$.

By using formula 245.00 in [8], from (3.1), we obtain a periodic blow up wave solution

$\varphi=\varphi_{1}+A-\frac{2 A}{1-\operatorname{cn}(\sqrt{2 A} \xi, k)}$,

where $A=\sqrt{\left(\frac{\varphi_{2}+\overline{\varphi_{2}}}{2}-\varphi_{1}\right)^{2}-\frac{\left(\varphi_{2}-\overline{\varphi_{2}}\right)^{2}}{4}}, k=\sqrt{\frac{2\left(A+\varphi_{1}\right)-\left(\varphi_{2}+\overline{\varphi_{2}}\right)}{4 A}}$ is the modulus of Jacobian elliptic function.

(2) When $f(\varphi)=-\left(\varphi-\varphi_{-}\right)^{2}\left(\varphi-\varphi_{1}\right)$, and $\varphi \leq \varphi_{-}$or $\varphi_{-}<\varphi \leq \varphi_{1}$, then $f(\varphi) \geq 0$.

Substituting $f(\varphi)$ into (2.4) and integrating it.

If $\varphi \leq \varphi_{-}$, we have 
$\int_{-\infty}^{\varphi} \frac{1}{\left(\varphi_{-}-t\right) \sqrt{\varphi_{1}-t}} d t=\int_{0}^{\xi} \sqrt{2} d t$

From (3.3), we obtain a blow up wave solution

$\varphi=\varphi_{-}-\left(\varphi_{1}-\varphi_{-}\right) \sinh ^{-2}\left(\frac{\sqrt{2\left(\varphi_{1}-\varphi_{-}\right)}}{2} \xi\right)$.

If $\varphi_{-}<\varphi \leq \varphi_{1}$, we have

$\int_{\varphi}^{\varphi_{1}} \frac{1}{\left(t-\varphi_{-}\right) \sqrt{\varphi_{1}-t}} d t=\int_{\xi}^{0} \sqrt{2} d t$

From (3.5), we obtain a solitary wave solution

$\varphi=\varphi_{-}+\left(\varphi_{1}-\varphi_{-}\right) \cosh ^{-2}\left(\frac{\sqrt{2\left(\varphi_{1}-\varphi_{-}\right)}}{2} \xi\right)$.

(3) When $f(\varphi)=-\left(\varphi-\varphi_{1}\right)\left(\varphi-\varphi_{2}\right)\left(\varphi-\varphi_{3}\right)$, and $\varphi \leq \varphi_{1}$ or $\varphi_{2} \leq \varphi<\varphi_{3}$, then $f(\varphi) \geq 0$.

Substituting $f(\varphi)$ into (2.4) and integrating it.

If $\varphi \leq \varphi_{1}$, we have

$\int_{-\infty}^{\varphi} \frac{1}{\sqrt{\left(\varphi_{3}-t\right)\left(\varphi_{2}-t\right)\left(\varphi_{1}-t\right)}} d t=\int_{0}^{\xi} \sqrt{2} d t$.

By using formula 231.00 in [8], from (3.7), we obtain a periodic blow up wave solution

$\varphi=\varphi_{3}-\left(\varphi_{3}-\varphi_{1}\right) \operatorname{sn}^{-2}\left(\frac{\sqrt{2\left(\varphi_{3}-\varphi_{1}\right)}}{2} \xi, k\right)$.

If $\varphi_{2} \leq \varphi<\varphi_{3}$, we have

$\int_{\varphi_{2}}^{\varphi} \frac{1}{\sqrt{\left(\varphi_{3}-t\right)\left(t-\varphi_{2}\right)\left(t-\varphi_{1}\right)}} d t=\int_{0}^{\xi} \sqrt{2} d t$

By using formula 236.00 in [8], from (3.7), we obtain a periodic wave solution

$\varphi=\varphi_{3}-\left(\varphi_{3}-\varphi_{2}\right) \operatorname{sn}^{2}\left(\frac{\sqrt{2\left(\varphi_{3}-\varphi_{1}\right)}}{2} \xi, k\right)$,

where $k=\sqrt{\frac{\varphi_{3}-\varphi_{2}}{\varphi_{3}-\varphi_{1}}}$ is the modulus of Jacobian elliptic function.

(4) When $f(\varphi)=-\left(\varphi-\varphi_{1}\right)\left(\varphi-\varphi_{+}\right)^{2}$, and $\varphi \leq \varphi_{1}$, then $f(\varphi) \geq 0$.

Substituting $f(\varphi)$ into (2.4) and integrating it, we have

$\int_{-\infty}^{\varphi} \frac{1}{\left(\varphi_{+}-t\right) \sqrt{\varphi_{1}-t}} d t=\int_{0}^{\xi} \sqrt{2} d t$.

From (3.11), we obtain a periodic wave solution

$\varphi=\varphi_{+}-\left(\varphi_{+}-\varphi_{1}\right) \csc ^{2}\left(\frac{\sqrt{2\left(\varphi_{+}-\varphi_{1}\right)}}{2} \xi\right)$.

Here we complete the proof of Proposition.

\section{Conclusion}

In this paper, we obtained some exact traveling wave solutions of KPI by using the theory of dynamical systems. We draw their plane graphs. Through studying shape of traveling waves, we shown that the traveling waves of KPI 
contain periodic blow-up waves (see Fig.1 (a,b,c)), blow-up wave (see Fig.1 (e)), smooth periodic waves (see Fig.1 (d)) and solitary wave (see Fig.1 (f)). Compare the previous results, periodic blow-up waves and blow-up wave are new.

\section{References}

[1] B.B.Kadomtsev,V.I.Petviashvili, On the stability of solitary waves in weakly dispersive media. Sov Phys Dokl 15 (1970), 539-541.

[2] M. Khalfallah, New exact traveling wave solutions of the $(3+1)$ dimensional Kadomtsev-Petviashvili (KP) equation. Commun Nonlinear Sci Numer Simul 14 (2009), 1169-75.

[3] I. Dmitry Sinelshchikov, Comment on: New exact traveling wave solutions of the $(3+1)$-dimensional Kadomtsev-Petviashvili (KP) equation. Commun Nonlinear Sci Numer Simul 15 (2010), 3235-6.

[4] Z.R. Liu , B. Guo, Peridic blow-up solutions and their limit forms for the generalized Camassa-Holm equation. Progress in Natural Sci 18 (2008), 259-66.

[5] S.L. Xie , L. Wang, Compacton and generalized kink wave solutions of the CH-DP equation. Appl Math Comput 215 (2010), 4028-4039.

[6] S.L. Xie, Q. Lin, B. Gao, Periodic and Solitary Travelling-Wave Solutions of a CH-DP Equation. Commun Nonlinear Sci Numer Simul 16 (2011), 3941-48

[7] S.L. Xie , L. Wang, Y.Z. Zhang, Explicit and implicit solutions of a generalized Camassa-Holm Kadomtsev-Petviashvili equation. Commun Nonlinear Sci Numer Simul 17 (2012), 1130-41.

[8] P.F. Byrd, M.D. Friedman, Handbook of elliptic integrals for engineers and scientists. Berlin: Springer; 1971.

\section{Received: March 9, 2014}

